\title{
Cris rituels, auloi et tambourins. Paysage sonore et identités dans les Bacchantes d'Euripide
}

\author{
Ritual shouts, auloi and drums. Soundscape and identities in Euripides' Bacchae
}

\author{
Georgios Christodoulou \\ Universitè Lille, Francia \\ georgebm95@yahoo.com
}

Charles Delattre

Universitè Lille, Francia

charles.delattre@univ-lille.fr

https://orcid.org/0000-0001-6702-6887

\begin{abstract}
RÉSUMÉ:
Cris rituels, auloí et tambourins sont mentionnés à plusieurs reprises dans le texte des Bacchantes d'Euripide. La pièce crée ainsi un univers sonore fictif, un tissu de références auditives qui se superpose à la musique réellement perçue par les spectateurs, dans le contexte de la première performance aux Dionysies à Athènes. Notre étude articule ces deux espaces sonores, en analysant en particulier le rôle de l'aulós. Cet instrument présente en effet une apparente contradiction: il est ce qui donne rythme et mélodie au festival athénien, mais il est aussi l'un des emblèmes du culte étranger. Nous montrons comment la configuration des sonorités propres aux Bacchantes multiplie les références sonores, et crée un espace sonore qui est un espace de médiation et de conciliation des identités.
\end{abstract}

Mots CLÉs: Tragédie, Paysage sonore, Fiction, Performance, Euripide.

\begin{abstract}
:
The text of Euripides' Bacchae mentions several times ritual shouts, auloi and drums. The theater play thus creates a fictitious sound universe, a fabric of auditory references that overlaps with the music actually perceived by the audience, in the context of the first performance at the Dionysia in Athens. Our study articulates these two sonorous spaces, by analyzing in particular the role of the aulós. This instrument indeed presents an apparent contradiction: it is what gives rhythm and melody to the Athenian festival, but it is also one of the emblems of the foreign cult. We show how the configuration of the sounds in the Bacchae multiplies the sound references, and creates a soundscape where identities are mediated and reconciled.
\end{abstract}

KEYWORDS: Tragedy, Soundscape, Fiction, Performance, Euripides.

Les Bacchantes sont une des dernières tragédies composées par Euripide, pendant son séjour en Macédoine, et auraient été représentées à Athènes à titre posthume, d'après certains commentaires antiques, ${ }^{1}$ tout en remportant le premier prix. ${ }^{2}$ Et il s'agit sans conteste de l'une des tragédies athéniennes les plus énigmatiques et les plus difficiles à interpréter dans le contexte du rituel des Dionysies: la pièce en performance confronte un culte civique athénien, consacré à Dionysos, et la représentation d'un culte, également consacré à Dionysos, mais présenté à la fois comme barbare et non civique. Dionysos, dieu d'Athènes autant qu'un autre, est représenté sur scène comme un dieu venu de l'étranger, même s'il s'agit pour lui de revenir sur la terre de ses ancêtres. Dans ce va-et-vient entre l'ici et l'ailleurs, le Grec et l'étranger, le cadre rituel et scénique et le contexte de l'intrigue, ${ }^{3}$ se joue la thématique de l'altérité, si 
finement analysée par J.-P. Vernant: ${ }^{4}$ dans ce dédoublement entre rituel local et représentation d'un culte exotique ${ }^{5}$ passe l'ambiguïté de Dionysos, figure du même et de l'autre.

La représentation de la pièce ajoute à cette thématique une dimension visuelle particulière, avec les jeux de masques propres au théâtre antique, ${ }^{6}$ et l'identification par les personnages d'un «étranger» ${ }^{7}$ qui se révèle finalement être le dieu même. ${ }^{8}$ Mais il ne faut pas négliger ce que peut apporter une étude du «paysage sonore» (soundscape) de la pièce. Cette notion, qui remonte au compositeur Raymond Murray Schafer à la fin des années 1960, ${ }^{9}$ fait du sonore un analogue du visuel, ${ }^{10}$ un univers que l'on peut décrire, voire inventorier, de façon à s'approcher de l'expérience sensible ${ }^{11}$ que constituait une performance dramatique. Or les Bacchantes représentent un exemple particulièrement riche et complexe d'univers sonore, en particulier dans la première partie de la pièce, comme l'indiquent les diverses allusions compilées dans le tableau suivant. ${ }^{12}$

\begin{tabular}{|c|c|c|c|}
\hline $\begin{array}{l}\text { DIVISIONS DES } \\
\text { BACCHANTES }\end{array}$ & VERS & LOCUTEUR & CONTENU \\
\hline Prologue & $58-61$ & Dionysos & $\begin{array}{l}\text { exhortation au choeur à lever et frapper les } \\
\text { tambourins phrygiens }\end{array}$ \\
\hline \multirow{2}{*}{ Párodos } & $120-34$ & choeur & $\begin{array}{l}\text { mythe étiologique de l'invention du tympanon; } \\
\text { melange avec l'aulós phrygien et l'évohé bachique }\end{array}$ \\
\hline & $155-65$ & $\begin{array}{l}\text { Dionysos projeté } \\
\text { (= choeur) }\end{array}$ & $\begin{array}{l}\text { exhortation au choeur à chanter et à jouer des } \\
\text { tambourins et de l'aulós }\end{array}$ \\
\hline Épisode I & $\varnothing$ & & \\
\hline stásimon I & $378-81$ & choeur & $\begin{array}{l}\text { Dionysos riant et conducteur du thiase, au son de } \\
\text { l'aulós }\end{array}$ \\
\hline Épisode II & $511-4$ & Penthée & $\begin{array}{l}\text { menace d'arrêter la frappe des tambourins par le } \\
\text { choeur }\end{array}$ \\
\hline stásimon II & $560-4$ & choeur & cithare d'Orphée \\
\hline \multirow{2}{*}{ Ëpisode III } & $686-8$ & messager $n^{e} 1$ & $\begin{array}{l}\text { réfutation de l'ivresse des bacchantes thébaines } \\
\text { prises par le vin et le son de l'aulós }\end{array}$ \\
\hline & $783-4$ & Penthée & $\begin{array}{l}\text { métaphore musicale (l'archer fait résonner la } \\
\text { corde de l'arc) }\end{array}$ \\
\hline stásimon III & $\varnothing$ & & \\
\hline Épisode IV & $951-2$ & $\begin{array}{l}\text { Ettranger } \\
\text { (Dionysos) }\end{array}$ & référence aux sifflements de la syrirx de Pan \\
\hline stásimon IV & $\varnothing$ & & \\
\hline \multirow[b]{2}{*}{ Ëpisode V } & 1034 & choeur & auto-référence au chant barbare \\
\hline & $1056-7$ & messager $n^{\circ} 2$ & $\begin{array}{l}\text { référence au chant bachique des bacchantes } \\
\text { thébaines }\end{array}$ \\
\hline stasimon V & $\varnothing$ & & \\
\hline Exodos & $\varnothing$ & & \\
\hline
\end{tabular}

Au-delà des mots, les cris apportent une tonalité particulière, non pas les exclamations qui expriment l'infortune, le désespoir ou la douleur, comme on en trouve dans la plupart des tragédies attiques

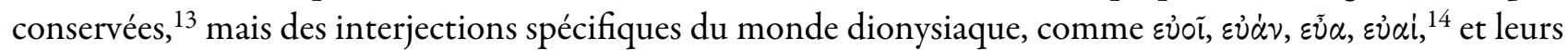
dérivés, le verbe $\varepsilon \dot{u} \dot{d} \zeta \omega$, à l'actif ou au moyen, le substantif $\varepsilon \dot{v} \dot{\alpha} \sigma \mu \alpha \tau \alpha$, et $\varepsilon \dot{v} ı \circ$, entendu soit comme surnom de Dionysos, soit comme adjectif. Au cours de la párodos, le chœur évoque Dionysos, revêtu de la nébride sacrée et pourchassant le bouc pour l'égorger (v. 135-138): il est celui qui «mène ses thiases dans les monts de Phrygie et de Lydie», ${ }^{15}$ une description ponctuée par un ev̉oĩ (v. 141) qui est proféré avec puissance par les adoratrices du dieu et qui peut être entendu à double sens par les spectateurs athéniens. En effet, la 
profération du cri «évohé», tout comme celui de ses dérivés dans les chants du chœur, enrichit l'effet rituel du drame: le cri s'explique comme adresse à Dionysos de la part des femmes lydiennes qui composent le chœur, mais renvoie aussi, au moins comme allusion, à l'ensemble de la fête des Dionysia au sein desquelles la performance se déroule. Et, dans le contexte précis de la description faite par les bacchantes du dieu Dionysos, on peut même y voir un cri poussé par Dionysos lui-même, comme si le dieu lançait l'interjection rituelle reprise par le chœur et légitimait ainsi le cri rituel dionysiaque.

Il en va de même des autres occurrences du cri «évohé», y compris sous ses formes dérivées: lorsque les bacchantes exposent que chacune d'elle «célèbre Bacchios», non pas «en l'évoquant», mais «en l'évohant», si l'on veut bien accepter ce jeu de mots, ${ }^{16}$ ou qu'elles répondent au messager qu'elles «l'évohent», ${ }^{17}$ elles font surgir ce cri rituel, non pas dans l'espace scénique, mais sous la forme d'une allusion qui résonne en écho, sinon dans la perception directe, au moins dans l'imagination auditive du public. L'effet est d'autant plus flagrant que le début de la parodos multiplie les termes qui invitent les spectateurs à redéfinir l'ensemble du chant, qu'il s'agisse d'une adaptation de la formule invitant à faire silence au début d'une pratique rituelle ${ }^{18}$ ou du verbe v́ $\mu \nu \varepsilon \iota \nu$ qui fait de ce passage l'équivalent d'un chant de célébration. ${ }^{19}$

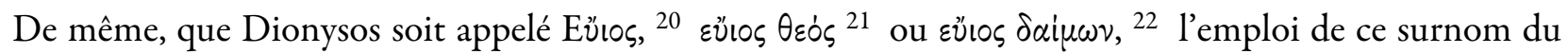
dieu ou du qualificatif correspondant crée des ambiguités sémantiques: ce dieu est caractérisé par la clameur d'évohé, il est le dieu de l'évohé et son surnom fait aussi surgir ce cri rituel, directement ou par réminiscence. ${ }^{23}$ L'analyse des deux occurrences du substantif $\varepsilon \dot{u} \dot{\sigma} \sigma \mu \alpha \tau \alpha$ dans la pièce conduit aux mêmes conclusions: le chœur emploie ce terme dans la párodos pour qualifier non directement son propre chant, mais celui des Bacchantes en général, quand elles célèbrent le dieu. ${ }^{24}$ Difficile cependant de ne pas établir avec cette désignation distanciée un lien direct avec que représente le chœur aux yeux du public, à savoir un ensemble de Bacchantes célébrant Dionysos. Même si le chant du chœur n'avait pas le rendu, la ligne mélodique, la définition même d'un chant rituel en l'honneur de Dionysos, pris qu'il était dans les contraintes musicales propres au genre tragique, il pouvait en susciter l'impression par suggestion. ${ }^{25}$

Les deux occurrences de $\varepsilon \dot{u} \dot{\alpha} \sigma \mu \alpha \tau \alpha$ sont elles-mêmes accompagnées de jeux sonores qui viennent compléter le dispositif et augmenter la complexité sonore, même si celle-ci n'est que suggérée. La deuxième occurrence sert en effet de toile de fond à un cri retentissant de Dionysos lui-même, qui incite les

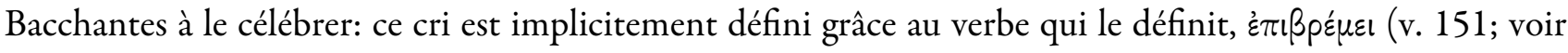
aussi v. 162), comme un «grondement sourd» analogue à celui du tonnerre, qui fait surgir en même temps dans l'espace sonore, comme une glose, l'un des noms de Dionysos, Bromios, «le Grondant», désigné comme tel au v. 115. Et à cela s'ajoute l'évocation des tambourins, qui accompagnent le chant ${ }^{26}$ de leur «sourd grondement». ${ }^{27}$ Ainsi le même son qualifie-t-il l'instrument, le cri du dieu et le dieu lui-même, dans un chant qui, pour être rigoureusement organisé du point de vue de la métrique, ne fait pas moins surgir l'idée du tapage, du vacarme et de la dissonance. ${ }^{28}$

La mention des tambourins associés au culte dionysiaque correspond à une évolution notable du dernier quart du Ve s.: alors qu'ils semblent liés principalement au culte de la Mère phrygienne avant cette époque, ${ }^{29}$ ils passent au main des Bacchantes, à la fois dans les textes ${ }^{30}$ et l'iconographie, où ils remplacent par exemple les krótala que l'on trouve sur les vases attiques depuis la deuxième moitié du VIe s. ${ }^{31}$ Ces tympana sont un tambour à main, généralement tenu dans la main gauche et joué avec les doigts ou la paume de la main droite. ${ }^{32} \mathrm{Si}$ l'on accepte leur présence sur scène, ${ }^{33}$ ils renforçaient rythmiquement les chants et les cris rituels proférés dans les différents stásima par le chœur des Bacchantes et contribuaient, par le vacarme qu'ils produisaient, à une sensation de frénésie. ${ }^{34}$ Et même s'ils n'étaient qu'un objet du discours, et non un instrument joué sur l 'orchéstra, ils faisaient partie de ces éléments considérés désormais comme significatifs du culte dionysiaque dont la suggestion donnait à entendre ce que l'oreille du public ne percevait pas forcément dans la réalité de la performance. Ils contribuaient ainsi à la définition du culte comme étranger à la cité grecque et contrastaient spectaculairement -c'est-à-dire, ici, auditivement-avec la musique qu'accompagnent les célébrations d'un rituel civique. 
La première occurrence de $\varepsilon \dot{\alpha} \sigma \mu \alpha \tau \alpha$ dans la párodos est, si possible, encore plus remarquable que la seconde, car elle accompagne non un déchaînement sonore suggéré par le texte, et éventuellement performé sur scène, mais un court récit étiologique qui remonte aux origines du tambourin en mêlant le culte dionysiaque à des références complémentaires (v. 120-134). Le vacarme, si l'on peut dire, n'est pas sonore, mais thématique, par a ccumulation de plusieurs séries de termes qui se superposent et se confondent. Tout entière contenue dans la deuxième antistrophe, cette évocation commence avec Zeus enfant, dans l'antre de Crète. ${ }^{35}$ Les Courètes qui a ccompagnent l'enfance de Zeus sont associés -ou identifiés?- aux Corybantes, à qui est attribuée l'invention d'un «orbe tendu de cuir», ${ }^{36}$ le premier tambourin, ${ }^{37}$ qu'ils remettent ensuite à Mère Rhéa pour rythmer les «chants d'évohé» ${ }^{38}$ des Bacchantes. Mais il faut encore une intervention, celle des Satyres, pour que le tambourin devienne pleinement l'instrument des danses 39 consacrées à Dionysos. Sont ainsi énumérés et partiellement confondus les Courètes et les Corybantes, la Crète et la Phrygie, ce qui renvoie à une équation bien connue qui réunit Rhéa et Cybèle, mais aussi plus largement culte de la Mère des dieux et culte de Dionysos, voire Phrygie et Lydie comme terres de référence. Le motif de la transmission sert de fil conducteur aux quinze vers de l'antistrophe, ${ }^{40}$ mais cette armature narrative sert aussi un processus d'identification généralisé: au chœur qui chante ce passage se superpose, à l'arrière-plan visuel et sonore dans l'imagination du public, les Bacchantes mentionnées par le texte qui constituent le cortège dionysiaque en général, les Satyres, et enfin les Corybantes et les Courètes euxmêmes. Le temps des origines, celui de l'enfance de Zeus, le temps de la constitution du cortège dionysiaque, le temps de l'intrigue elle-même et celui de la c élébration s'associent pour faire résonner un tambourin dont la voix mime le grondement même du dieu que l es Dionysies célèbrent. L'ensemble du chant est comme une épiphanie sonore permise par le rituel athénien.

D'autres instruments enrichissent le paysage sonore sur un mode mineur. Dans le deuxième stásimon, il est ainsi question «d'Orphée jouant de la cithare», ${ }^{41}$ sans référence directe ni à la performance chorale ni au culte dionysiaque -à moins que la mort d'Orphée aux mains de Bacchantes, bien connue au moins depuis les Bassarai d'Eschyle, ne suscite un contraste tragique. Lorsque Penthée donne l'ordre à ses archers de 1 ivrer bataille aux Bacchantes, il les désigne comme «ceux dont la main fait vibrer la corde d'un arc»: ${ }^{42}$ le verbe $\psi \alpha \dot{\alpha} \lambda \omega \omega$ s'applique d'abord à la vibration imposée par le doigt sur une corde d'instrument, la métaphore reposant sur l'analogie bien connue entre l'arc et la lyre. ${ }^{43}$ Enfin, la syrinx associée à Pan est rapidement mentionnée dans le quatrième épisode, dans un passage où Dionysos se moque cruellement de Penthée et l'invite à épargner «les séjours de Pan où l'on entend siffler sa syrinx», ${ }^{44}$ entraînant un court instant l'imagination du spectateur vers des paysages sonores qui ne sont plus ceux de l'oreibasie, mais ceux des rythmes et mélodies champêtres.

Dans ce paysage sonore complexe, fait d'aller-et-retour entre la réalité de la performance, l'intrigue de la pièce et les différents arrières-plans mobilisés par le texte, l'aulós joue un rôle particulier. Tout comme le tambourin, l'aulós est en effet régulièrement défini comme un instrument propre aux cultes orgiastiques, associé à la danse extatique et à la musique sauvage venue d'Orient, plus spécifiquement de Phrygie. ${ }^{45}$ Aristote le définit d'ailleurs comme un instrument proprement «orgiastique», incapable de susciter un comportement «éthique», ${ }^{46}$ et Platon en fait le seul instrument susceptible de déclencher une véritable possession. ${ }^{47}$

Dans les passages de la párodos où résonnent le cri de l'évohé et le grondement des tambourins, le timbre pénétrant de l'aulós se fait également entendre, dans le même univers sonore où le nom de l'instrument suffit à évoquer le son qui peut être le sien: «le souffle des auloí, doux cri de Phrygie, se mélange en accordant ses tons aux actes des bacchants», ${ }^{48}$ quand les Corybantes remettent le tambourin récemment inventé à Mère Rhéa, et «les éclats du saint lotos grondent» à la fin de l'épode, ${ }^{49}$ imitant la voix du dieu comme le font 
les tambourins quelques vers plus haut, dans l'épiphanie sonore généralisée que nous décelons à la fin de la párodos.

Rien ne semble distinguer l'aulós du tambourin ou des cris rituels dans l'ensemble de la párodos. Une différence essentielle apparaîtcependant pour cet instrument dans le premier stásimon (v. 370-433), qui reconduit et inverse certaines des caractéristiques sonores associées au culte dionysiaque. Ce chant du chœur, divisé en deux paires de strophes, est un commentaire lyrique à l'épisode précédent, les strophes qui le composent étant directement liées à la situation dramatique: la première strophe (v. 370-85) dénonce les propos agressifs que vient de tenir Penthée, et la deuxième strophe (v. 402-15) exprime le désir ardent du chœur de s'évader vers des terres d'où le rite dionysiasque n'est pas proscrit comme à Thèbes. Dionysos reste le sujet du chant, mais, même s'il reste ce Bromios qui gronde (v. 375), il est désormais le dieu du banquet partagé, de la joie et des couronnes. «Thiase et danses» sont toujours mentionnés, ${ }^{50}$ mais se déploient dans une atmosphère qui n'est plus celle des courses folles sur le Cithéron, mais celle des rituels sociaux au cœur de la cité. La première strophe construit un nouveau paysage sonore, celui où résonnent le «rire» qu'accompagnent les auloi, ${ }^{51}$ et qui tourne au «silence reposant» ${ }^{52}$ qu'apporte le «sommeil». ${ }^{53}$

Dionysos est ici le vin du cratère, le breuvage réparateur qui réunit les hommes, associé à Aphrodite (v. 402-405) et aux Muses (v. 409-411).

Ce changement brusque de ton, voire de tonalité musicale, n'est pas un cas unique dans la pièce. De fait, il est même thématisé à l'apparition d'Agavé sur scène, lorsque se retrouvent pour la première fois en présence les femmes de Thèbes qui célébraient le culte de Dionysos sur le Cithéron et les Bacchantes étrangères qui le célébraient dans l'espace de l'orchéstra (1165-1199). À la folie d'Agavé et de ses compagnes, meurtrières de Penthée, s'oppose la parole du chœur, certes exultant de la victoire de Dionysos sur son ennemi, mais c lairvoyant et conscient des enjeux.

À ce dédoublement des valeurs de l'aulós, entre célébration d'un culte dont l'origine est attribué à la Phrygie et à la Lydie, et festivités athéniennes intégrées dans les pratiques de sociabilité, en correspond un autre, celui de l'aulós mentionné dans l'intrigue et celui qu'entend le public, pour le coup sans que le doute soit permis. On sait bien en effet que l'aulós est l'instrument qui accompagne la tragédie, qui en rythme le chant et en organise la mélodie. ${ }^{54}$ Cela est dû peut-être au fait que l'aulós produit un son au timbre pénétrant, facilement audible depuis les gradins du théâtre. ${ }^{55}$ Ce n'est cependant pas forcément cet argument technique qui peut justifier l'emploi de l'aulós, mais les usages qui sont les siens et les connotations qui lui sont associées. Il existe par exemple un débat dans l'école aristotélicienne sur les valeurs respectives de l'aulós et de la lyre. Dun côté, ils contribuent tous les deux à la perception claire d'une mélodie, et se suffisent à eux-mêmes, $s$ ans besoin d'instruments supplémentaires (Ps. Aristote, Problèmes 19.9). Mais l'aulós est meilleur que la lyre pour accompagner un chant, car il se fond mieux avec la voix et couvre les erreurs du chanteur, alors que la lyre les met en évidence (Ps. Aristote, Problèmes 19.43).

Cette affinité entre l'aulós et la voix humaine est exploitée par Euripide lui-même, par exemple dans l'Oreste où la voix des femmes du chœur est comparée au «souffle dans la tige du roseau léger de la syrinx». ${ }^{56}$ Contrairement à la lyre ou à la cithare, qui se distinguent en apportant leur propre sonorité, l'aulós a cette capacité à rejoindre au plus près la voix humaine: à la différence des instruments à cordes pincées ou percutées, le son qu'il produit est issu d'un souffle, comme celui qui parcourt la gorge et à partir duquel les mots peuvent se former. Lyre et cithare sont par ailleurs susceptibles, tout autant que l'aulós, de produire une mélodie, mais il leur manque l'impact que produit le rythme, non pas celui des tambourins, avec leurs percussions caractéristiques, mais celui qui accompagne l'émission continue de la voix. Ce rythme, c'est celui des longues et des brèves sur lesquelles se module le chant, qu'il s'agisse de celui que produit la scène tragique ou de celui qui résonne en péan avant et pendant la bataille. ${ }^{57}$

L'aulète, qui se tient sur l'orchéstra en costume tragique, est l'ordonnateur de la performance: c'est sur son rythme que se règlent les choreutes, à la fois pour produire leur chant et pour évoluer dans l'espace, quel 
qu'ait été le type de danse ou de mouvements expressifs qu'ils aient effectué. L'emméleia, un terme qui peut renvoyer aussi bien aux unités rythmiques et mélodiques du chant qu'aux membres du corps qui se règlent sur elles (mélos), ${ }^{58}$ dit bien l'harmonie requise sur la scène tragique, c'est-à-dire l'étroite correspondance entre l'instrument musical tenu par l'aulète et les corps qui se meuvent et chantent dans l'espace régulé de l'orchéstra.

Il ne suffit donc pas de reconnaître que l'aulós est autant l'instrument nécessaire à la performance du théâtre tragique que celui du culte dionysiaque. Dans le paysage sonore perçu par le public comme dans celui construit par le texte, l'aulós joue un même rôle identificatoire, il est l'analogue d'un embrayeur qui, dans une stratégie de communication verbale, renvoie à une situation d'énonciation et participe à l'actualisation d'un énoncé. Le son de l'aulós est ce qui signifie, dans l'espace de l'orchéstra, le cadre rituel dans lequel l'ensemble du dispositif scénique prend place, il rattache les spectateurs à l'bic et nunc de la célébration rituelle. Ses effets mélodiques et rythmiques sont du côté des personnes, acteurs et choreutes, et non des personnages, en ceci qu'ils se conforment à un cadre culturel prédéfini, celui du théâtre athénien. D'un autre côté, les mentions des auloí sont des évocations suggestives qui construisent des espaces de représentation sonore et qui donnent l'illusion au public d'entendre autre chose que ce qu'il entend dans la réalité. Le spectateur à la fois voit un acteur sur scène et se représente un personnage, ce qui est une définition élémentaire du théâtre; mais il est aussi auditeur, et en tant que tel, est soumis à un procédé de duplication sonore identique à celui qui s'applique à son champ de vision.

Par rapport aux tambourins et aux cris rituels, l'aulós bénéficie d'un avantage complémentaire: il est physiquement présent dans la réalité du spectacle, tout en participant à l'évocation du champ sonore dans le cadre de la fiction dramatique. Ce dédoublement de l'aulós est en fait singulier, et lui donne un pouvoir d'articulation remarquable: il est en effet ce qui affirme la juxtaposition de la fiction dramatique avec la réalité cultuelle. En ce sens, il est ce qui rend manifeste l'analogue d'un code-switching, c'est-à-dire le passage d'un système d'interprétation à l'autre. ${ }^{59}$ De même qu'une langue peut intégrer dans un énoncé non seulement des mots empruntés à une autre langue, mais des éléments syntaxiquement régulés qui, de ce fait, appartiennent en même temps à deux systèmes linguistiques, le dispositif dramatique se fonde sur l'association et la complémentarité d'une perception sensorielle ancrée dans la réalité et d'une représentation visuelle et sonore qui ne se déploie que dans le monde intérieur d'un spectateur-auditeur. L'aulós, présent dans les deux systèmes, est l'un des points de jonction où l'équilibre dramatique se joue. Il assure la production de l'univers sonore scénique et la construction d'un exotisme sensoriel fantasmé. ${ }^{60}$

L'une des originalités des Bacchantes, c'est non seulement d'avoir utilisé l'aulós comme une clé du dispositif général, mais d'en avoir fait en quelque sorte le commentaire. On se rappelle en effet que le Dionysos célébré dans la párodos au son des auloí et des tambourins est lydien et phrygien, tandis que le dieu des banquets chanté dans le premier stásimon est athénien. On peut donc dire que l'aulós est à la fois phrygien et grec, il est à la fois ce qui guide les mouvements et les cris des Bacchantes et ce qui règle l'ordonnancement du banquet, à condition de se rappeler que cette double définition se fait dans l'espace sonore représenté, et non perçu, et s'articule donc avec un troisième type d'aulós, celui dont joue l'aulète dans l'orchéstra pour accompagner cette même évocation.

S'il y a jeu d'altérité, polarité entre l'ici et l'ailleurs, le Grec et l'autre dans les Bacchantes, ce jeu est démultiplié et se déploie dans différentes dimensions, et surtout il est constamment en action. Si l'attention se porte un instant sur l'aulós de l'espace scénique, la mention d'un aulós dionysiaque en modifie la perception en introduisant la suggestion d'une autre sonorité qui se superpose à celle que produit l'aulète, voire s'y confond même ou l'annule. Mais cet effet ne dure qu'un instant, et l'auditeur réajuste son oreille à son environnement sonore, pour être pris ensuite par un nouvel effet, cri rituel ou allusion au tambourin, qui reconduit l'imagination vers de nouvelles voies (ou voix). Les identités phrygiennes, lydiennes, grecques et athéniennes, malgré leur apparente solidité, sont soumises à un flux permanent en se greffant sur ces objets eux-mêmes labiles que sont les tambourins et les auloí. Dionysos lui-même est-il lydien, phrygien, 
grec, thébain, ou athénien, tantôt l'un tantôt l'autre, ou plutôt tout cela à la fois, en tension perpétuelle avec lui-même? Le dieu qui a adopté la forme d'un étranger, comme un vêtement, qui pourra en dire l'identité véritable?

Le premier stasimon est exemplaire de cette tension générale, qui est comme un jeu de miroirs: le banquet grec où règne Dionysos est célébré par les Bacchantes lydiennes, au son d'un aulós dont les notes résonnent dans un terroir athénien dont Thèbes est la contrepartie dans la fiction dramatique. En dernier ressort, cette Phrygie et cette Lydie dont il est question font intégralement partie de l'espace athénien, quoiqu'elles soient prétendues étrangères. On sait bien que l'exotisme n'est qu'une stratégie de représentation qui occulte les identités revendiquées par l'autre, au profit des identités construites par celui qui tient le discours de l'exotisme. Les notes de l'aulós dionysiaque des Bacchantes, parce qu'elles sont suggérées et non jouées, parce qu'elles sont rêvées et non perçues, n'ont pas à justifier leur statut d'étrangères par des détails historiquement avérés. Mais leur exotisme est d'autant plus efficace qu'elles résonnent en contrepoint avec les notes que joue l'aulète: c'est dans ce contraste sonore paradoxal, entre perception et imagination, que s'élaborent à la fois la définition de Dionysos et celle du théâtre athénien. L'un des enjeux des Bacchantes, c'est d'avoir thématisé et transposé sur le mode sonore cette articulation du spectacle tragique.

\section{REFERENCIAS}

Adams, J. N. (2003). Bilingualism and the Latin Language. Cambridge: Cambridge University Press.

Bierl, A. (2013). Maenadism as self-referential chorality in Euripides' Bacchae. En R. Gagné y M. Govers-Hopman (Eds.), Choral Mediations in Greek Tragedy (pp. 211-226). Cambridge: Cambridge University Press.

Biraud, M. (2010). Les interjections du thétre grec antique. tude sémantique et pragmatique. Louvain: Peeters.

Bond, G. W. (1963). Euripides. Hypsipyle. Oxford: Oxford University Press.

Bouchon, R., Brillet-Dubois, P. y Le Meur-Weissman, N. (2012). Introduction. En R. Bouchon, P. Brillet-Dubois y N. Le Meur-Weissman (Eds.), Hymnes de la Grèce antique. Approches littéraires et historiques (pp. 9-18). Lyon: Maison de l'Orient et de la Méditerranée -Jean Pouilloux.

Calame, Cl. (2017). La tragédie grecque comme performance musicale et rituelle. Voix et danses chorales. En P. Katuszewski y M. Manca (Eds.), Théâtres du geste, du jeu et de la voix (p. 11-26). Bordeaux: Presses universitaires de Bordeaux.

Crawley, A. E. (1931). Dress, Drink and Drums. Further Studies of savages and sex. London: Methuen \& Co.

Dickey, E. (2018). What is a Loanword? The case of Latin borrowings and codeswitches in Ancient Greek. LL, 17(1), 7-36.

Di Giulio, A. M. (1991). The Frame Drum as a Dionysian Symbol in Scenes on Apulian Pottery. RIdIM/RCMI Newsletter 16(1), 2-7.

Dodds,E. R. (1960). Euripides Bacchae, edited with introduction and commentary. Oxford: Oxford University Press.

Gurd, S. A. (2016). Dissonance. Auditory Aesthetics in Ancient Greece. New York: Fordham University Press.

Hall, J. M. (1997). Ethnic Identity in Greek Antiquity. Cambridge: Cambridge University Press.

Hall, J. M. (2002). Hellenicity. Between Ethnicity and Culture. Chicago: The University of Chicago Press.

Henrichs, A. (1993). 'He Has a God in Him'. Human and Divine in the Modern View of Dionysus. En Th. H. Carpenter y Chr. A. Faraone (Eds.), Masks of Dionysus (pp. 13-43). Ithaca, London: Cornell University Press.

Henrichs, A. (2011). Göttliche Präsenz als Differenz. Dionysosals epiphanischer Gott. En R. Schlesier (Ed.), $A$ Different God? Dionysos and Ancient Polytheism (pp. 105-116). Berlin, Boston: de Gruyter.

Henrichs, A. (2019). Göttliche Präsenz als Differenz. Dionysosals epiphanischer Gott. En H. Yunis (Ed.), Albert Henrichs. Greek myth and religion. Collected Papers 2 (pp. 451-464). Berlin, Boston: De Gruyter.

Ingold, T. (2007). Against Soundscape. En A. Carlyle (Ed.), Autumn Leaves. Sound and the Environment in Artistic Practice (pp. 10-13). Paris: Double Entendre. 
Ingold, T. (2011). Four objections to the concept of soundscape. En Being Alive. Essays on movement, knowledge and description (pp. 136-139). London, New York: Routledge.

Landels, J. G. (1999). Music in Ancient Greece and Rome. London, New York: Routledge.

Le Meur-Weissman, N. (2012). Les dithyrambes de Pindare et de Bacchylide sont-ils des hymnes?. En R. Bouchon, P. Brillet-Dubois y N. Le Meur-Weissman (Eds.), Hymnes de la Gréce antique. Approches littéraires et historiques (pp. 79-103). Lyon: Maison de l'Orient et de la Méditerranée -Jean Pouilloux.

Monbrun, Ph. (2007). Les voix d'Apollon. L'arc, la lyre et les oracles. Rennes: Presses universitaires de Rennes.

Nordgren, L. (2015). Greek Interjections. Syntax, Semantics and Pragmatics. Berlin y Boston: De Gruyter.

Perrot, S. (2015). Pour une archéologie des expériences sonores en Grèce antique. En S. Emerit, S. Perrot y A. Vincent (Eds.), Le paysage sonore de l'Antiquité. Méthodologie, historiographie et perspectives (pp. 175-208). Châtillon: Institut français d'archéologie orientale.

Saï, S. (1984). Grecs ou barbares dans le théâtre d'Euripide: la fin des différences?. Ktema, 9, 27-53.

Saïd, S. (1991). Introduction. En S. Saïd (Ed.), EAAHNI $M M O \Sigma$. Quelques jalons pour une histoire de l'identité grecque (pp. 3-7). Leyden: Brill.

Schafer, R. M. (1993). The Soundscape. Our Sonic Environment and the Tuning of the World. New York: Destiny Books. Taplin, O. (1978). Greek Tragedy in Action. London, New York: Routledge.

Vincent, A. (2015). Paysage sonore et sciences sociales. Sonorités, sens, histoire. En S. Emerit, S. Perrot y A. Vincent (Eds.), Le paysage sonore de l'Antiquité. Méthodologie, historiographie et perspectives (pp. 9-40). Châtillon: Institut français d'archéologie orientale.

Weiss, N. A. (2018). The Music of Tragedy. Performance and Imagination in Euripidean Theater. Berkeley: University of California Press.

West, M. L. (1992). Ancient Greek Music. Oxford: Oxford University Press.

\section{Notes}

1 Schol. Aristophane, Grenouilles 67; cf. Vita Euripidis 1.29. Certaines allusions des Bacchantes (v. 201-203, 270-271, 274 et suiv., 890 et suiv.) semblent spécifiquement destinées aux oreilles athéniennes. À noter que nous utilisons l'édition établie par E. R. Dodds en 1960.

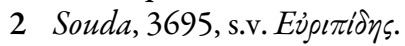

3 Pour une présentation générale de la confrontation entre fiction dramatique et performance scénique, avec attention particulière portée au chœur, voir par exemple Calame (2017) ainsi que Bierl (2013, p. 211-213) pour une application de ce dualisme aux chants choraux des Bacchantes.

4 Il faut cependant noter ici que l'altérité n'est pas strictement bipolaire, puisque Dionysos, dans le prologue où il énumère les terres qu'il a traversées (v. 10-24) inclut non seulement la Lydie, la Phrygie, la Bactriane, le pays des Mèdes, l'Arabie, que l'on peut opposer globalement au «pays grec» ('E $\lambda \hat{\alpha} \varsigma$ ), mais aussi l'Asie, où vivent «Grecs mêlés aux

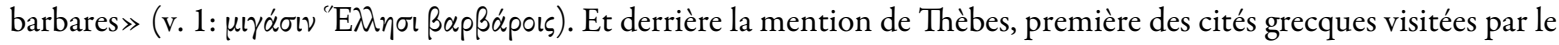
dieu et séjour de sa famille maternelle (v. 23-27), se profile également Athènes, qui le célèbre. C'est donc une construction à plusieurs étages, où la désignation de ce qui est «grec» n'est pas forcément unifié: voir à ce sujet les remarques de Saï (1984), généralisées dans Saï (1991, p. 4), et amplifiées depuis par de nombreuses études, à commencer par celles de Hall $(1997,2002)$. Voir aussi les nuances apportées au modèle de l'altérité par Henrichs (1993, p. 31-39), ainsi que sa définition de Dionysos comme dieu épiphanique (Henrichs, $2011=$ Henrichs, 2019).

5 À noter que le terme $\beta \alpha ́ p \beta \alpha \rho o \varsigma$ revient à plusieurs reprises dans les Bacchantes pour désigner le terroir d'origine des participants au culte (v. 56-57; 1054) ou les Bacchantes elles-mêmes (v. 482 ç; 604; 1033; 1057; 1334), mais aussi Paphos de Chypre (v. 406-408) et les territoires d'Asie où vivent également des Grecs (v. 18). Il est employé aussi bien par Dionysos (v. 56-57; 482; 1054-1057; 1334) que par les Bacchantes (v. 1033; 406-408), et de façon plus négative par Cadmos (v. 1054-1057), mais jamais par Penthée. Même si une connotation péjorative peut être associée au terme, il est possible ici que la dimension linguistique et sonore attachée au terme motive son emploi.

6 N'est pas seulement en cause le recours aux masques, mais le problème théologique de la représentation du dieu, à la fois pour les spectateurs et dans l'intrigue: comment en effet reconnaître un dieu qui a «échangé sa forme divine pour celle

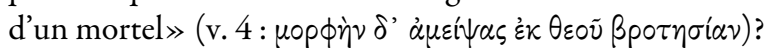

7 v. 233: 乡́ंvos. 
8 Voir en particulier les v. 1340-1347, qui articulent les deux identités de Dionysos, l'étranger et le dieu, avec le début et la fin de l'intrigue.

9 Schafer (1993). Voir Vincent, 2015, p. 9: «Un paysage sonore est la représentation par un individu ou un groupe d'individus, dont les sens sont le produit d'une construction sociale historiquement datée et contextualisée, d'un ensemble d'événements culturels sonores entendus en un lieu et un temps historique déterminés».

10 Perrot (2015, p. 176).

11 Nous considérons ici que le son est un phénomène d'expérience, suivant les mots d'Ingold (2007, p. 11), repris dans Ingold (2011, p. 136): «Sound is, in my view, neither mental nor material, but a phenomenon of experience -that is of our immersion in, and commingling with, the world in which we find ourselves.»

12 Le tableau montre bien que les chants du chœur concentrent la plupart des allusions à l'univers sonore, comme on pouvait s'y attendre, mais il illustre aussi que tous les stasima ne sont pas concernés, ce qui semble indiquer une évolution du paysage sonore au fur et à mesure que la pièce se déroule. Par ailleurs nous laisserons ici de côté la difficile question de la relation entre cet univers sonore et celui de la Nea Mousikè, pour nous attacher ici seulement à sa configuration.

13 Voir Biraud (2010), complété par Nordgren (2015).

14 En dehors des Bacchantes, voir par exemple, pour घủoĩ, Aristophane, Lysistrata 1294 et Sophocle, Trachiniennes 219;

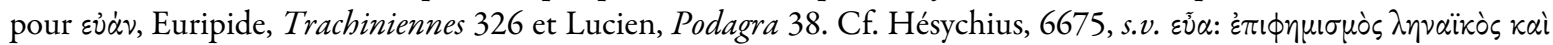

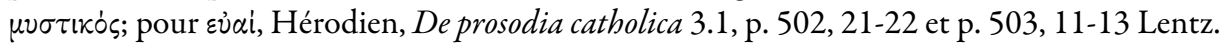

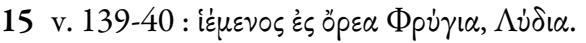

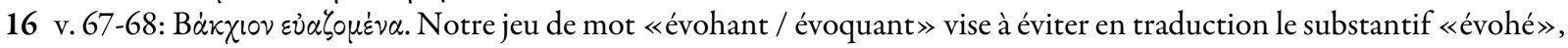
car le recours au verbe empêche justement la profération du cri tout en le suggérant.

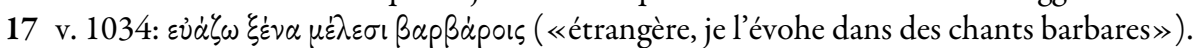

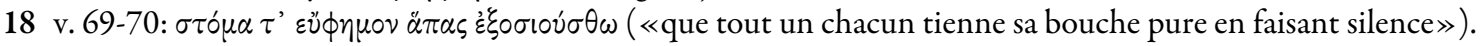

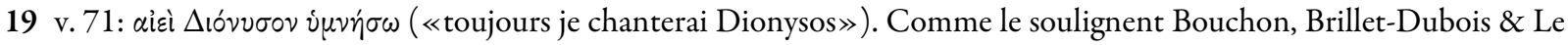
Meur-Weissman (2012, p. 12), un hymne est «un discours qui définit la nature et les prérogatives du dieu», plutôt qu'un genre poétique particulier; voir aussi Le Meur-Weissman (2012, p. 82-83).

20 v. 566 et 579 .

21 v. 1167.

22 v. 413.

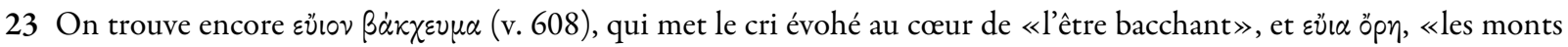
de l'évohé» (v. 791), qui renvoient au Cithéron dans la pièce et au motif de l'oreibasie. Pour des montagnes portant ce nom, voir Pausanias, 4.31.4 (à proximité de Messène) et Polybe, 2.65 (près de Sparte).

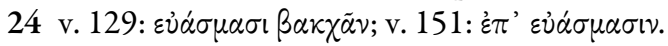

25 Weiss (2018) parle de imaginative projection pour ce type de suggestion, en particulier lorsque le chœur incite le spectateur à l'identifier sa performance à d'autres performances rituelles et musicales.

26 v. 155: $\mu \varepsilon \lambda \pi \varepsilon \tau \varepsilon$.

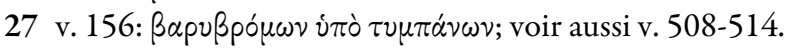

28 Voir Gurd, 2016, sur cette notion.

29 Voir par exemple Hérodote 4.76.

30 Pour ne s'en tenir qu'à Euripide, voir Fr 586 Kannicht; Cyclope 63-72; 205; avec une transposition à Aphrodite, Hélène, 1347.

31 Pour les krotala que tiennent des Bacchantes, voir par exemple un psykter du Louvre (F 321) des années 520-510 (LIMC, Mainades 329), ou une kylix attique à figures rouges de Munich (Antikensammlung 2645) du Peintre de Brygos des années 480. Le tambourin est bien présent dans la céramique apulienne: voir l'étude de Di Giulio (1991).

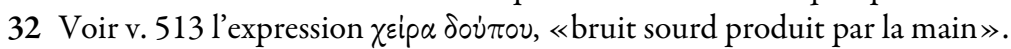

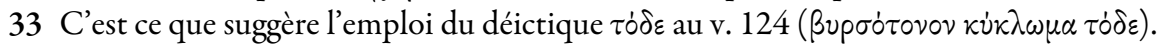

34 Voir Landels (1999, p. 81-2); West (1992, p. 122-6).

35 Ce premier élément est cohérent avec Hésiode, Théogonie 453-93, tandis que le reste ne l'est pas.

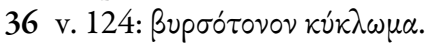

37 Ce n'est qu'à partir de Callimaque, Hymne à Zeus 1.51-53, qu'apparaît l'idée que les Courètes frappaient sur des boucliers en bronze pour couvrir les vagissements de Zeus. Cette version est répandue à l'époque impériale: on la trouve par exemple chez Ps. Apollodore, Bibliothéque 1.1.7, et elle est traitée à deux reprises par Nonnos de Panopolis, Dionysiaques 13.135-6 et 153-158. Les cymbala évoquées par Virgile, Géorgiques 4.149-52, désignent un instrument de bronze circulaire associé au monde dionysiaque et sont une métaphore des boucliers sonores. Le lien entre le tambourin et le bouclier, tous deux objet circulaire, est évident, mais l'état de notre documentation ne permet pas de conclure où réside l'originalité d'Euripide: le tambourin est-il le résultat d'un jeu qui transpose le motif du bouclier dans un cadre musical? Ou bien est-ce Callimaque qui assure cette transposition, cette fois du tambourin au bouclier? Il est même fort possible que le tambourin, chez Euripide, et le bouclier, chez Callimaque soient tous les deux des innovations. 


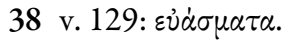

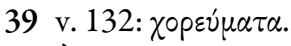

40 À noter que cette transmission reformule les propos de Dionysos lui-même dans le prologue: le dieu indique en effet que

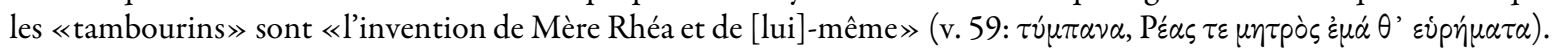
Nous ne suivons pas Dodds (1960, p. 83-4), qui voit dans la première version une forme plus ancienne, et dans la deuxième une forme ancienne, peut-être de l'initiative d'Euripide. Les deux formulations jouent de façon différenciée sur la double articulation Phrygie-Lydie et Asie Mineure-pays grec.

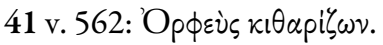

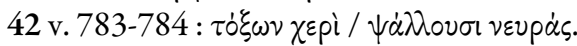

43 Pour la signification musicale du verbe $\psi \alpha \dot{\alpha} \lambda \omega$, voir par exemple Hérodote, 1.155; Aristophane, Cavaliers 522; Platon, Lysias 209b; Aristote, Problèmes 19.23.1. Sur les correspondances entre l'arc et les instruments à cordes, voir Monbrun (2007, p. 31-178).

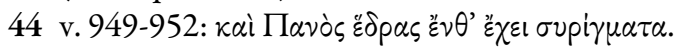

45 Voir Euripide, Électre 1351; Ménandre, Théophoroumene 27 et suiv. Pour les tambourins, voir West (1992, p. 122-126); sur le caractère orgiastique des tambourins, voir Crawley (1931, p. 248) et suiv., ainsi qu'Euripide, Héraclès furieux 889; Hélène 1347; Cyclope 65 et 205; Aristophane, Guêpes 119; Lysistrata 3 et 338.

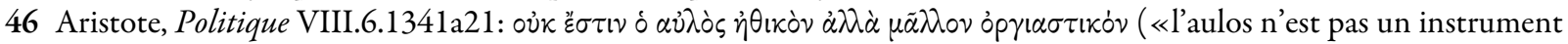
tant éthique qu'orgiastique»).

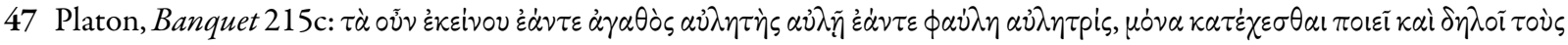

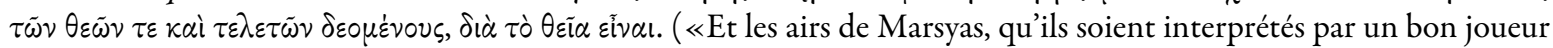
d'aulós ou par une joueuse minable, ce sont les seuls capables de nous mettre dans un état de possession et, parce que ce sont des airs divins, de faire voir quels sont ceux qui ont besoin des dieux et d'initiations»; trad. L. Brisson).

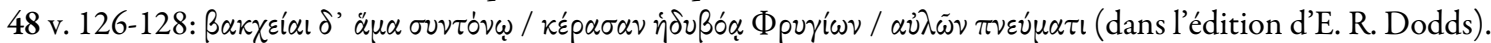

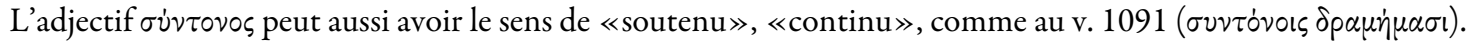

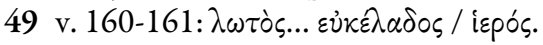

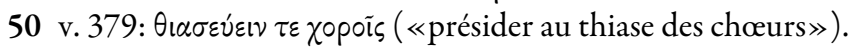

51 v. 380: $\mu \varepsilon \tau \dot{\alpha} \tau$ ' $\alpha \dot{u} \lambda \circ \tilde{v} \gamma \varepsilon \lambda \alpha \dot{\sigma} \sigma \alpha \mathrm{l}$ («rire avec l'aulos).

52 v. 381: $\dot{\alpha} \pi \circ \pi \alpha \tilde{v} \sigma \alpha i$ $\tau \varepsilon \mu \varepsilon p i ́ \mu \nu \alpha \varsigma$ (《mettre fin aux soucis»).

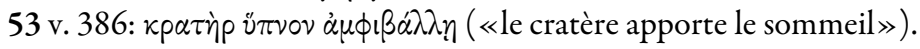

54 D'autres instruments ont peut-être été utilisés, si l'on utilise le texte pour reconstituer non un paysage sonore imaginaire,

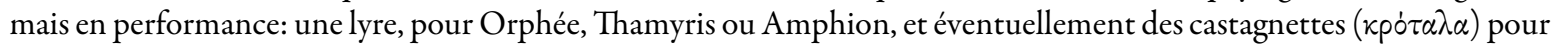
Hypsipyle (voir Euripide, Hypsipyle Fr 769 Kannicht avec le commentaire de Bond 1963, p. 139-140). Au sujet des divers instrument dans des tragédies spécifiques, voir West (1992, p. 351, n. 110).

55 Voir l'emploi de l'adjectif $\varepsilon \dot{\kappa} \varepsilon \hat{\lambda} \alpha \delta \circ \varsigma$, «retentissant», v. 160, et l'expression $\lambda \omega \tau \sigma \tilde{~} \psi o ́ \phi \omega$, , «le bruit du lôtos» (équivalent de l'aulos), v. 687.

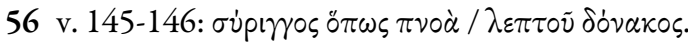

57 Un exemple frappant de l'utilisation de l'aulos pour accompagner le combat est fournie par une olpé de style polychrome protocorinthien, des années 640 à 630 avant notre ère, le «Vase Chigi», conservé à la Villa Giulia (22 679).

58 Voir Taplin (1978, p. 8-9), et Landels (1999, p. 110).

59 Le code-switching, intégration temporaire d'un terme dans une langue étrangère, se distingue linguistiquement de l'emprunt, intégration constante et partagée d'un terme dans une langue étrangère, même si la distinction n'est pas toujours aisée à établir (voir à ce propos les remarques de Dickey, 2018). Les conséquences du code-switching sont analysées par Adams (2003, p. 297-416), pour le cas du latin incluant dans son énonciation des termes grecs.

60 Sur le rôle du code-switching dans la construction d'un exotisme, voir Adams (2003, p. 403-405). 